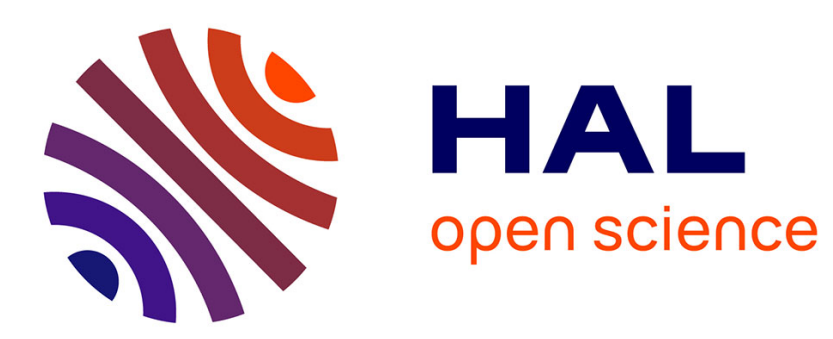

\title{
Energetic neutral atom fluxes from the heliosheath varying with the activity phase of the solar cycle
}

\author{
H.-J. Fahr, K. Scherer
}

\section{To cite this version:}

H.-J. Fahr, K. Scherer. Energetic neutral atom fluxes from the heliosheath varying with the activity phase of the solar cycle. Astrophysics and Space Sciences Transactions, 2004, 1 (1), pp.3-15. hal00297469

\section{HAL Id: hal-00297469 \\ https://hal.science/hal-00297469}

Submitted on 30 Nov 2004

HAL is a multi-disciplinary open access archive for the deposit and dissemination of scientific research documents, whether they are published or not. The documents may come from teaching and research institutions in France or abroad, or from public or private research centers.
L'archive ouverte pluridisciplinaire HAL, est destinée au dépôt et à la diffusion de documents scientifiques de niveau recherche, publiés ou non, émanant des établissements d'enseignement et de recherche français ou étrangers, des laboratoires publics ou privés. 


\title{
Energetic neutral atom fluxes from the heliosheath varying with the activity phase of the solar cycle
}

\author{
H.-J. Fahr and K. Scherer \\ Institut für Astrophysik und Extraterrestrische Forschung der Universität Bonn, Auf dem Hügel 71, D-53121 Bonn, Germany
}

Received: 6. June 2004 - Revised: 15 October 2004 - Accepted: 21 November 2004 - Published: 30 November 2004

\begin{abstract}
Energetic neutral atoms (ENA's) which are produced in the heliospheric interface can be used to remotely investigate this highly interesting, but poorly known region acting as buffer against the interstellar plasma. To optimally take advantage from this very promising observational technique, based on ENA flux measurements, one needs to know the spectral production rate of the ENA's in the heliosheath and about the foreground ENA contaminations originating in the inner heliosphere, e.g. inside the termination shock. Heliosheath ENA's with energies of about a few $\mathrm{KeV}$ originate from charge exchange reactions of $\mathrm{H}$-atoms both with the shocked solar wind protons and with pick-up protons downstream of the termination shock. Inner heliospheric ENA's detectable at the Earth can only originate from $\mathrm{H}$ charge exchange reactions with pick-up protons. In this article we calculate on the basis of the five-fluid Bonn model the timedependent ENA fluxes that are connected with these three sources and compare them. It will be demonstrated that the heliosheath ENA fluxes dominate from nearly all directions over the inner heliospheric ENA fluxes, though the best chances to disentangle the contributions appear in upwind and downwind direction at specific periods of the solar activity cycle.
\end{abstract}

\section{Introduction to the heliospheric ENA-productions}

The location and geometry of the solar wind termination shock and the downstream plasma interface configuration has been a subject of intensive theoretical investigations (for a review see Zank, 1999; Izmodenov et al., 2000; Fahr et al., 2000; Zank and Müller, 2003, etc.). Nevertheless the physics, geometry and scales of these configurations are still very much a matter of debate, especially since relevant observations up to now do not allow for consistent interpretations. Perhaps most recently some signatures of the solar wind termination shock structure and of the post-shock plasma flow

Correspondence to: kscherer@astro.uni-bonn.de) might have been identified for the first time in the running particle flux measurements carried out by the distant NASA space-probe VOYAGER-1 (see Krimigis et al., 2003; McDonald et al., 2003). Though the interpretation of these recent observations still are fairly controversial (see McDonald et al., 2003; Burlaga et al., 2003) it is considered as a serious possibility that in fact VOYAGER-1 may have made the first step into the post-shock plasma region of the heliosphere. Even if so, there are many open questions left, not only concerning the location of the shock and its local and temporal variability, but also concerning the physics and structure of this shock. While the multi-fluid character of this shock transition was already clearly recognized (Zank et al., 1993; Chalov and Fahr, 1994, 1995, 1997; LeRoux and Fichtner, 1997; Kausch and Fahr, 1997; Fahr et al., 2000), the prediction of the exact transition properties of the downstream plasma flow are fairly qualitative and unreliable in the degree in which open parameters are used that up to now lack clear observations. This partly discouraging fact one should keep in mind even when the relevant dynamic multifluid interaction processes are meanwhile nicely understood and modelled and seem to present trustworthy representations of global heliospheric interface structures.

As was already discussed in Gruntman et al. (2001) the ENAs can be used to remotely image the outer heliosphere. This explains why presently there is a strong wish amongst heliospheric scientists that all these theoretical predictions should soon find their reliable basis in relevant observational data. With the forthcoming IBEX-mission (Interstellar Boundary EXplorer) (see McComas et al., 2004) the promising idea comes that many details of the predicted heliospheric structures could appear reflected in earth-observable spectral fluxes of energetic neutral atoms serving as messengers of the physical secrets of the distant heliospheric plasma sites. Already in the recent past the method of using "energetic neutral atom"- imaging (ENA-imaging) has been proposed to remotely study active plasma regions at planets, comets and in the outer heliosphere. While ENA-diagnostics of the earth magnetosphere and ionosphere has already suc- 
cessfully been carried out with instruments onboard the satellite IMAGE (Burch, 2003), the ENA-diagnostics of largely extended plasma regions like especially the heliospheric interface is naturally complicated by the problem to reliably deconvolute line-of sight informations into local informations, especially since here time-dependent phenomena play non-negligible roles (for reviews see Williams et al., 1992; Gruntman, 1997).

This complication for the use of the above mentioned ENA-technique at application to heliospheric diagnostics has been studied and modelled in recent works by Scherer and Fahr (2002, 2003a). Especially in Scherer and Fahr (2003b) the time-dependent ENA-production in the heliospheric multifluid interface under the action of solar-cycle induced solar wind momentum flow variations has been studied. As shown by these authors fairly complicated, fully time-dependent and non-periodic reactions of the whole interface system lead to ENA-fluxes variable with time and direction. In addition to this complication another difficulty to carry out ENA-diagnostics of the outer heliosphere arises from the fact that there exists a contaminating foreground ENA-radiation, since even in the supersonic solar wind region there exist pick-up ions (PUIs) with sunwards directed velocities. These can transform into energetic $\mathrm{H}$-atoms by charge exchange processes with the penetrating neutral $\mathrm{H}$-atoms from the interstellar wind and hit a detector at $1 \mathrm{AU}$. In the heliosheath hot solar wind protons, pick-up ions and anomalous cosmic rays (ACR) are present which at the occasion of becoming neutralized by interstellar H-atoms can produce KeV ENA's targeted to a detector at Earth (Hilchenbach et al., 1998). While production rates of ACR-ENAs in the heliosheath are fairly small and negligible below $100 \mathrm{KeV}$ (see especially Fig. 3 in Czechowski et al., 2001b), ENA's from decharged solar wind protons and PUIs are competing in fluxes. In addition also decharged PUI's from the region of supersonic solar wind, i.e. the inner heliosphere, upon the occasion of becoming decharged by interplanetary $\mathrm{H}$-atoms, have to be taken into account as contamining foregrounds.

In the following paper we shall demonstrate that IBEX will do fertile science of the heliospheric interface by observing the ENA-fluxes and their time-variabilities. For that purpose we also intend to take into account pick-up ions (PUI's) which upon becoming decharged by neutral H-atoms produce energetic ENA's both in the inner and in the outer heliosphere.

\section{The underlying time-dependent interface model}

Normal protons and PUI's which are appearing in the inner and outer heliosphere before they become converted into the corresponding ENA components need to be described by a consistent interface model which takes into account both the consistent interactions of all dynamically and thermodynamically relevant fluids and the time-dependent reaction of the whole plasma interface under the action of solar-cyclic variations of the inner solar wind ram pressure. In our case this consistent modelling is based on a hydrodynamic fivefluid model developed by Fahr et al. (2000) taking into account the consistent hydrodynamic interaction of low energetic fluids like protons and $\mathrm{H}$-atoms, middle-energetic fluids like PUI's, and high-energetic fluids like anomalous cosmic rays and galactic cosmic rays. This model more recently has been expanded to a fully time-dependent modelling in order to treat the solar-cyclic breathing of the heliospheric interface (Scherer and Fahr, 2002, 2003a,b) and reveals the multimodal and multiperiodic reactions of all interface properties. Alternative hydrodynamic or semi-kinetic models for time-dependent reactions of the heliospheric structures under solar-cyclic solar wind ram pressure variations have been presented by Zank and Müller (2003) or by Izmodenov and Malama (2004). While Scherer and Fahr (2002, 2003a,b) and Zank and Müller (2003) find clearly pronounced irreversible switch-on phenomena connected with irreversible dislocations of the heliospheric boundaries and the accumulation of dissipated thermal energy in the heliosheath plasma, Izmodenov and Malama (2004) only obtain a system with monoperiodic variations of all plasma properties and with oscillations of all boundaries around the stationary locations of solar cycle average ram pressure.

In our hydrodynamical model, called the Bonn model, a consistent coupling of five different fluids, namely protons, $\mathrm{H}$-atoms, PUI's, anomalous cosmic rays (ACR's) and galactic cosmic rays (GCR's) is treated. This model which originally was run for stationary boundary conditions more recently has also been used to treat time-dependent boundary conditions by Scherer and Fahr (2002, 2003a,b), primarily for the purpose to study time-variabilities of the heliospheric interface under the action of solar cyclically variable inner boundary conditions due to the periodically variable inner solar wind ram pressure. This advanced modelling of the interface shall now also be used here to deliver the hydrodynamic properties of protons and PUI's at all places of the inner and outer heliosphere at all events of time during consecutive solar cycles. With these properties made available we then start to calculate the originating time-dependent ENA fluxes according to the method described below.

\section{Theoretical approach and calculations}

As basis of our calculations by which we determine the spectral ENA fluxes originating in the heliospheric interface we again here use the same procedure as already introduced and applied by Scherer and Fahr (2003b). Since now, however, we aim at including also PUI-induced ENA's, we have to add new terms to describe the additional contributions from PUIinduced ENA's. In these new terms simply the corresponding proton properties have to be replaced by analogous properties of the PUI's.

The local ENA production rates in the heliospheric interface are given by e.g. Scherer and Fahr (2003b) in the form:

$\Psi(\boldsymbol{r}, \boldsymbol{v}, \tau)=\left|n_{\mathrm{i}} f_{\mathrm{i}}(\boldsymbol{v}) n_{\mathrm{H}} \sigma_{\text {ex }}\left(v_{\text {rel }}\right) v_{\text {rel }}\right|_{\boldsymbol{r}, \tau}$ 
where $n_{\mathrm{i}}$ and $n_{\mathrm{H}}$ are the densities of the local protons $i=p$, of PUI's $i=$ pui, and of the H-atoms, respectively. $f_{\mathrm{i}}(\boldsymbol{v})$ is the proton/PUI velocity distribution function, $\sigma_{\mathrm{ex}}$ is the charge exchange cross section, and $v_{\text {rel }}$ is the mean relative velocity between H-atoms and protons/PUI's of velocity $\boldsymbol{v}$.

The newly introduced expression for the PUI-induced ENA production, i.e.:

$$
\Psi_{\text {pui }}(\boldsymbol{r}, \boldsymbol{v}, \tau)=\left|n_{\text {pui }} f_{\text {pui }}(\boldsymbol{v}) n_{\mathrm{H}} \sigma_{\text {ex }}\left(v_{\text {rel }}\right) v_{\text {rel }}(v)\right|_{\boldsymbol{r}, \tau},
$$

now describes the rate of ENA's produced from charge exchange reactions of H-atoms with PUI's in addition to those with protons. Here $v_{\text {rel }}$ is the mean relative velocity between H-atoms and PUI's of velocity $\boldsymbol{v}$ which in view of the fact that $v$ is equal to:

$$
\begin{aligned}
v_{\text {rel }} & =\sqrt{v^{2}+v_{H}^{2}-2 v_{H} v \cos \left(\boldsymbol{v}, \boldsymbol{v}_{H}\right)} \\
& \simeq v\left(1-\frac{v_{H}}{v}\right) \simeq v .
\end{aligned}
$$

and thus can be simply replaced by $v$.

\section{The PUI distribution function}

To carry out the above mentioned calculations of spectral PUI-induced ENA fluxes one of course needs to know the PUI distribution function both at places upstream and downstream the termination shock, because PUI- induced ENA's arriving at a detector near Earth are as well produced in the region of the supersonic solar wind as in that of the postshock subsonic solar wind. On the other hand, no contributions have to be considered in the region outside of the heliopause, since PUI's due to vanishing spatial diffusion practically are not transported out to this transheliopause region.

\subsection{Upstream PUI's}

First we shall give here the PUI distribution function $f_{\text {pui }}(r, v)$ inside the termination shock, i.e. in the region of the supersonic solar wind. As is well known PUI's in the inner heliosphere undergo various migration processes in phase-space, like injection, pitch angle scattering, convection, adiabatic cooling, and momentum diffusion as discussed and treated by Chalov and Fahr (1998). The combined effects of these processes in shaping the pitchangleisotropized function $f_{\text {pui }}(r, v)$ are adequately described by a PUI phase-space transport equation which has been solved by Chalov et al. $(1995,1997)$ yielding the distribution function $f_{\text {pui }}(r, v)$ in the solar wind rest frame. As shown by Fahr and Lay (2000) for standard turbulence conditions upstream of the termination shock their results can be represented in the following analytical form:

$$
\begin{aligned}
& f_{\text {pui }}^{\text {up }}(r, w)=\frac{2}{3} \frac{4 \pi^{3 / 2}}{3} \frac{C(x)^{3 / 2}}{\Gamma(3 / 2)} \\
& \cdot(1+w)^{\beta} \cdot \exp \left[-C(x)\left(w-w_{0}\right)^{\kappa}\right] \\
& =\frac{16 \pi}{9} C(x)^{3 / 2} \cdot(1+w)^{\beta} \cdot \exp \left[-C(x)\left(w-w_{0}\right)^{\kappa}\right]
\end{aligned}
$$

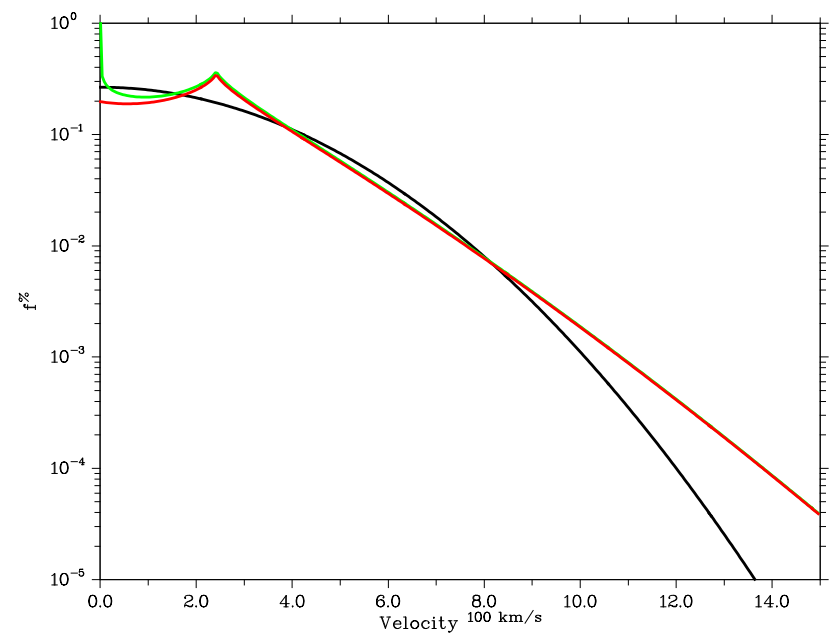

Fig. 1. For the region between the termination shock and the heliopause an example is shown for the normalized distribution functions of ENAs (Maxwellian, black) produced by the shocked solar wind protons and one of ENAs produced from PUIs ( $f_{\text {pui }}^{\text {up }}$, red), respectively. The green distribution function is the fit for large velocities obtained by Fahr and Lay (2000), while the red curve shows the continuation of this function to lower velocities.

and the local PUI density $n_{\text {pui }}(\boldsymbol{r})$ is given by:

$n_{\text {pui }}(\boldsymbol{r})=2 \pi \int_{0}^{\infty} f_{\text {pui }}^{\text {up }}(r, w) \sqrt{w} d w$

in the above equations, $x=r / r_{E}$ is the normalized solar distance where $r, R_{E}$ are in units of $\mathrm{AU}$, and $w=\left(v / V_{s}\right)^{2}$ is the squared PUI velocity normalized to the upstream solar wind bulk velocity $V_{s}$. The typical normalized PUI injection energy is denoted by $w_{0}=0.83$. Furthermore the quantities $\beta, \kappa$, and $C(x)$ are found as: $\beta=-(1 / 6) ; \kappa=(2 / 3)$; and $C(x)=0.442 \cdot x^{0.2} . \Gamma$ is the well known Gamma-function $(\Gamma(3 / 2)=1 / 2 \sqrt{\pi})$.To express the above spectral density in units of $\left[\mathrm{cm}^{-3} \mathrm{KeV}^{-1}\right]$ one simply has to multiply the above expression by the factor $\left[V_{s 0}^{2} / V_{s}^{2}\right]$, where the reference velocity $V_{s 0}$ is defined by: $(1 / 2) \mathrm{mV}_{s 0}^{2}=1 \mathrm{KeV}$. The term $w^{\beta}$ in the original fit by Fahr and Lay (2000) was replaced by $(1+w)^{\beta}$ which allows for a better continuation to small normalized PUI velocities $w<1$, but leaves the original distribution function unchanged for large values of $w$. Both distribution functions are shown in Fig. 1 together with the Maxwellian governing the solar wind proton-Hydrogen exchange processes.

\subsection{Downstream PUI's}

When arriving under an off-axis angle $\theta$ (which is unique in our axisymetrical model) at the termination shock at $x_{s}(\theta)$, PUI's locally undergo a rapid change in their distribution function $f_{\text {pui }}^{\text {up }}(r, v) \rightarrow f_{\text {pui }}^{\text {down }}(r, v)$ at the passage over the shock due to requirements of the Liouville theorem and the conserved magnetic invariant. This then leads to the fol- 
lowing function (see Fahr and Lay, 2000; Czechowski et al., 2001a):

$$
\begin{aligned}
f_{\mathrm{pui}}^{\text {down }}\left(x_{s}(\theta), w\right) & =\frac{2}{3} \frac{4 \pi^{3 / 2}}{3} s(\theta) \epsilon(\theta)^{-3 / 2} \frac{C\left(x_{s}\right)^{3 / 2}}{\Gamma(3 / 2)} \\
& \cdot\left(\frac{1+w}{s(\theta)}\right)^{\beta} \exp \left[-C\left(x_{s}\right)\left(\frac{w-w_{0}}{s(\theta)}\right)^{\kappa}\right] \\
= & \frac{16 \pi}{9} s(\theta) \epsilon(\theta)^{-3 / 2} C\left(x_{s}\right)^{2 / 2} \\
& \cdot\left(\frac{1+w}{s(\theta)}\right)^{\beta} \exp \left[-C\left(x_{s}\right)\left(\frac{w-w_{0}}{s(\theta)}\right)^{\kappa}\right]
\end{aligned}
$$

where it should be kept in mind that again here the variable $w$ has as normalization velocity the upstream solar wind velocity $V_{s}$, where $s(\theta)=\left(\rho_{\text {down }} / \rho_{\text {up }}\right)_{\theta}$ is the compression ratio at the shock, and where $\epsilon(\theta)$ defines the magnetic field compression ratio given by:

$$
\begin{aligned}
\epsilon(\theta) & =s(\theta) \sqrt{\frac{1+\frac{t g^{2} \xi}{s(\theta)^{2}}}{1+t g^{2} \xi}}=\sqrt{s^{2}(\theta)+\left(1-s^{2}(\theta)\right) \sin ^{2} \xi} \\
& \approx s(\theta)+\frac{1}{2} \frac{1-s^{2}(\theta)}{s(\theta)} \xi^{2}
\end{aligned}
$$

with $\xi$ being the angle between the upstream Archimedian magnetic field $\boldsymbol{B}_{\text {up }}$ and the local shock surface which is defined by (see Czechowski et al., 2001b):

$\operatorname{tg} \xi=\frac{r_{s}(\theta)}{\frac{\partial r_{s}}{\partial \theta}}$

The angle $\xi$ between the shock surface and magnetic field is usually much less the $\pi / 4$ and therefore we may neglect the second term in the Taylor-expansion and approximate Eq. (7) by:

$\epsilon(\theta) \approx s(\theta)$

\section{Evolution of the downstream PUI distribution}

For the extended region downstream of the termination shock we now do assume the following simplifications in our description of the distribution function $f_{\text {pui }}^{\text {down }}(\boldsymbol{r}, w)$ : We may start out from the stationary transport equation which was already used by Czechowski et al. (2001b) or Chalov et al. (2003) given in the following form:

$$
\begin{aligned}
\left(\boldsymbol{v}_{s} \cdot \nabla\right) & f_{\mathrm{pui}}^{\text {down }}-\nabla \cdot\left(\kappa_{r} \nabla f_{\mathrm{pui}}^{\text {down }}\right) \\
& -\frac{1}{v^{2}} \frac{\partial}{\partial v}\left(v^{2} \kappa_{v} \frac{\partial}{\partial v} f_{\mathrm{pui}}^{\text {down }}\right)+\frac{v}{3}\left(\nabla \cdot \boldsymbol{v}_{s}\right) \frac{\partial}{\partial v} f_{\mathrm{pui}}^{\text {down }} \\
= & Q_{\mathrm{pui}}-\beta_{\mathrm{ex}} f_{\mathrm{pui}}^{\text {down }}
\end{aligned}
$$

where the terms, read from left to right, describe convection, spatial diffusion, velocity-diffusion, adiabatic deceleration, PUI-injection and PUI-removal. Here the quantities $\kappa_{r}$ and $\kappa_{v}$ denote the spatial and the velocity diffusion coefficient, respectively. The quantity $\boldsymbol{v}_{s}$ is the local subsonic solar wind bulk velocity.
We first assume that spatial diffusion at the relatively low energies of the PUI's, as also done in the case of upstream PUI's, can be neglected here, i.e. $\kappa_{r} \simeq 0$. Also the adiabatic cooling connected with the divergence of the solar wind bulk velocity may be considered here as negligibly small, since in the highly subsonic, nearly incompressible downstream solar wind flow it turns out (see e.g. Fahr, 2000) that $\left(\nabla \cdot \boldsymbol{v}_{\boldsymbol{s}}\right)=-(1 / \rho)\left(\boldsymbol{v}_{s} \cdot \nabla\right) \rho \simeq 0$. Also momentum diffusion (i.e. Fermi-2 acceleration) may be considered as essentially negligible (i.e. $\kappa_{v} \simeq 0$ ), since preferentially turbulences with propagation vectors $k$ oriented towards the shock can be expected in the interface flow downstream of the shock (see McKenzie and Westphal, 1969). This fact, however, suppresses the Fermi-2 acceleration. Thus one is then left with a fairly simplified but, nevertheless nearly correct, PUI transport equation given by:

$\left(\boldsymbol{v}_{s} \cdot \nabla\right) f_{\text {pui }}^{\text {down }}=Q_{\text {pui }}-\beta_{\text {ex }} f_{\text {pui }}^{\text {down }}$

where the last term on the right side describes the PUI losses by charge exchange with $\mathrm{H}$-atoms of density $n_{H}$. Here the charge exchange rate is given by: $\beta_{\mathrm{ex}}=n_{H} \sigma_{\mathrm{ex}}(v) v$. The PUI injection term as given by Chalov et al. (2003) has the form:

$Q_{\text {pui }}=\beta_{\text {ex }} \frac{n_{\text {pui }}}{4 \pi v^{2}} \delta\left(v-v_{0}\right)$

where the injection velocity is given by:

$v_{0}=\sqrt{v_{H}^{2}+v_{s}^{2}-2 v_{H} v_{s} \cos (H, s)} \simeq O\left(v_{H}, v_{s}\right) \ll v$

Since typical values $v$ of downstream PUI's of interest are of the order of the upstream solar wind velocity $V_{s}$ or larger, it follows that PUI injections are only taking place at fairly low velocities $v_{0} \ll v$. When furthermore velocity diffusion, however, is not operating, then it means, that freshly injected new PUI's are simply sitting at these low velocities $v_{0}$ not influencing the rest of the spectral density $f_{\text {pui }}^{\text {down }}(\boldsymbol{r}, v)$. Consequently the above differential equation for $f_{\text {pui }}^{\text {down }}$, given by Eq. (10) can then in a fairly good approximation be solved in the following form:

$$
\begin{aligned}
& f_{\text {pui }}(\boldsymbol{r}(s(\theta)), w)=f_{\text {pui }}^{\text {down }}\left(x_{s}(\theta), w\right) \\
& \cdot \exp \left[-\int_{0}^{s(\theta)} \frac{n_{H} \sigma_{\mathrm{ex}}(v) V_{s} w}{v_{s}(\theta)} d s\right] \\
&=f_{\text {pui }}^{\text {down }}\left(x_{s}(\theta), w\right) \exp \left[-n_{H} \sigma_{\text {ex }}(v) V_{s} w \tau(s(\theta))\right]
\end{aligned}
$$

where the exponential term describes the extinction along the path of the PUIs and the travel time $\tau(s(\theta))$ is given by:

$\tau(s(\theta))=\int_{0}^{s(\theta)} \frac{d s}{v_{s}(\theta)}$

For an upper limit extinctions can be neglected. These are connected with ENA-productions further inwards on the line of sight and thus deplete the spectral intensity further out. Therefore, Eq. (14) can be approximated by the assumption:

$$
f_{\text {pui }}^{\text {sheath }}(r, v)=f_{\text {pui }}^{\text {down }}\left(x_{s}(\theta), v\right) \frac{n_{\text {pui }}(r, \theta)}{n_{\text {pui }}\left(r_{T S}\right), \theta}
$$




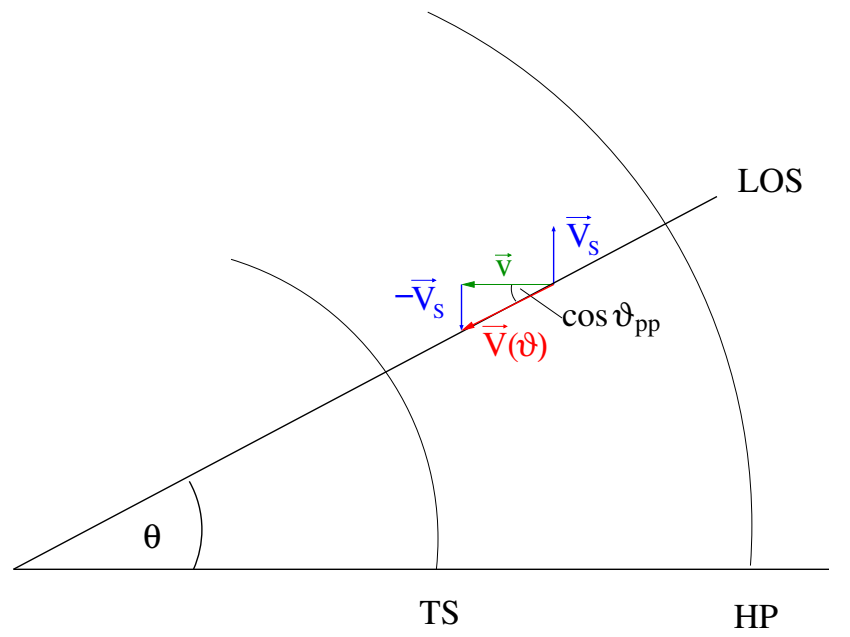

Fig. 2. Sketch of velocity vectors needed for the LOS integration.

A summation of the differential travel times e.g. along the stagnation line in a phase of the declining solar cycle with $V_{s}=600 \mathrm{~km} / \mathrm{s}$ at the termination shock $\left(r_{s}=82.3 \mathrm{AU}\right)$ and $v_{s}=0 \mathrm{~km} / \mathrm{s}$ at the heliopause $\left(r_{h}=151.5 \mathrm{AU}\right)$, yielding an average bulk velocity of $v_{s}=60 \mathrm{~km} / \mathrm{s}$, leads to the following travel time $\tau(s(0))\left(r_{h}-r_{s}\right) / v_{s}=1.07 \cdot 10^{8} \mathrm{~s} \approx 3.4$ years. The factor in the extinction $n_{H} \sigma_{\mathrm{ex}} \tau(s(0)) V_{s} \approx 9 \cdot \cdot 10^{-8} 1 / \mathrm{s}$. For the lowest energy of $0.2 \mathrm{KeV}$ PUI the extinction is of the order of 0.5 and for the largest energy of $35 \mathrm{KeV}$ in the order of $10^{-12}$. A more quantitative estimate of the resulting extinction losses is given in Sect. 8.

An example for the distribution responsible for the formation of ENAs from the shocked solar wind protons (a Maxwellian) is shown in Fig. 1 as the black curve while the distribution function $f_{\text {pui }}^{\text {up }}$ is plotted in red, the green curve shows the distribution function $f_{\text {pui }}^{\text {up }}$ from Fahr and Lay (2000), which is valid for large velocities. All distribution functions are normalized to 1 in the range between 0 and $35 \mathrm{KeV}$ and taken at solar minimum conditions.

\section{The line of sight collection}

So far we have discussed the local distribution functions. Now we need to select just that part from the distribution function describing ENA's which reach a detector at a specific position with a specific energy. In the axisymmetric Bonn model (Fahr et al., 2000; Scherer and Fahr, 2003a) the latitudinal grid size is $5^{\circ}$ which correspond to a detector with the same angular resolution. Selecting now a special line of sight with its origin at the Earth (i.e. practically identical with that to the Sun) and with an inclination of $\Theta$ with respect to the local interstellar medium (LISM) wind axis, that requires protons/PUIs of a special inclination $\cos \vartheta_{p p}$ with respect to the local proton bulk velocity $\boldsymbol{v}_{s}$ in order to gain appropriate $\mathrm{H}$-atoms which after creation fly into the wanted direction along this selected Line Of Sight (LOS) to finally reach the Earth (see Fig. 2). The requested velocity argument in the
Table 1. Energy intervals and time of flight, i.e. the maximum travel time taken into account, so that particles from $1500 \mathrm{AU}$ with a given mean energy can reach the detector.

\begin{tabular}{rrrc}
\hline \multicolumn{3}{c}{ kinetic energy $[\mathrm{KeV}]$} & max. time of \\
mean & lower & upper & flight [years] \\
\hline 0.2 & 0.1 & 0.3 & 35.0 \\
0.4 & 0.3 & 0.6 & 27.0 \\
0.8 & 0.6 & 0.9 & 19.0 \\
1.0 & 0.9 & 1.2 & 18.0 \\
1.5 & 1.2 & 2.0 & 14.0 \\
2.5 & 2.0 & 3.0 & 11.0 \\
3.5 & 3.0 & 4.7 & 9.0 \\
6.0 & 4.7 & 7.0 & 3.5 \\
8.0 & 7.0 & 10.0 & 2.3 \\
15.0 & 10.0 & 20.0 & 1.6 \\
35.0 & 25.0 & 50.0 & 0.8 \\
\hline
\end{tabular}

isotropic proton/PUI velocity distribution function hence is given by:

$v^{2}(\Theta)=\left(\boldsymbol{v}_{\boldsymbol{s}}-\boldsymbol{v}\right)^{2}=v_{s}^{2}+v^{2} \pm 2 v v_{s} \cos \vartheta_{\mathrm{pp}}$

where $\cos \vartheta_{p p}$, in order to meet the line of sight (LOS) - condition, has to fulfill the following condition:

$\cos \vartheta_{p p}=\cos \Theta \frac{v_{s, z}}{v_{s}}-\sin \Theta \frac{v_{s, x}}{v_{s}}$

where $v_{s, z}$ and $v_{s, x}$ are the components of $\boldsymbol{v}_{s}$ perpendicular and parallel to the inflow axis, respectively, which are delivered from our multifluid Bonn model.

Now the LOS-integrated H-ENA production yielding $\mathrm{H}$ atoms that move with the velocity $v(\Theta)$ along the line of sight up to their arrival at Earth at time $t$ is given by:

$$
\begin{aligned}
\Phi_{\mathrm{ENA}}(v(\Theta), \Theta, t)=\sum_{i} \int_{\mathrm{IB}}^{\mathrm{BS}} \Psi_{i}(\boldsymbol{r}, \boldsymbol{v}, \tau) d s \\
=\sum_{i} \int_{\mathrm{IB}}^{\mathrm{BS}}\left|n_{i} f_{i}(\boldsymbol{v}(\Theta)) n_{\mathrm{H}} \sigma_{\mathrm{ex}}\left(v_{\mathrm{rel}}\right) v_{\mathrm{rel}}\right|_{\boldsymbol{r}, \tau} d s
\end{aligned}
$$

where the retarded time $\tau=t-s / v(\Theta)$ takes into account the time of flight for an ENA particle of velocity $v(\Theta)$ along the distance $s$. The inner integration boundary $(I B)$ of the model is taken at $5 \mathrm{AU}$ (Scherer and Fahr, 2003b). The outer integration boundary is the bow shock $(B S)$, beyond which the ENA production is again negligible. The maximum time of flight is then given by $\tau_{\max }=L_{\max } / v(\Theta)$, where $L_{\max }$ is either the BS-distance or a cut-off distance in the tail region (see below).

In the tail region the particle may originate within distances of $L_{\max }=1500 \mathrm{AU}$ away from the Sun (Earth), if that distance is smaller than that to the bow shock, and ENA's still survive on their passage to the detector. Beyond $1500 \mathrm{AU}$ no 


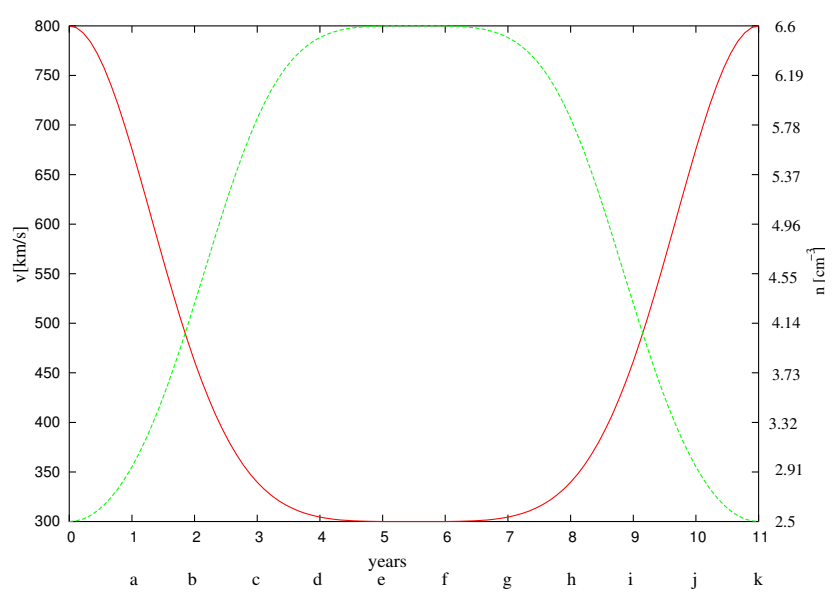

Fig. 3. The variations of the solar wind speed and density at the inner boundary with the solar cycle. The labels on the left $y$-axis give the variation of the solar wind speed (red curve), while that of the right hand side denotes the variation in the solar wind proton number density (green curve). The $x$-axis gives the time in years over one complete solar 11-year cycle, while the labels below the $x$-axis correspond to the labels given in Fig. (4) to Fig. (6).

further production relevant at the detector is assumed (for further discussion see Scherer and Fahr, 2003b).

In Table 1. the energy ranges are listed, which are displayed in Figs. 4 to 6.

\section{Solar cycle imprints onto the heliospheric interface}

To describe proton-induced or PUI-induced ENA fluxes arriving at the Earth at different time phases of the solar activity cycle, one primarily needs a detailed knowledge of the time-dependent properties of the background plasma flows, i.e. the solar wind and the interstellar plasma flow. PUI's are convected with these time-dependent flows and have time-dependent production rates. Neutral interstellar Hatoms are penetrating through these streaming plasmas and also thereby experience time-dependent losses and gains due to charge exchange reactions with the plasma constituents. In addition also energetic particles propagate through the plasma background in a diffusive-convective manner. To obtain the energy-averaged GCR (Galactic Cosmic Rays) and ACR (Anomalous Cosmic Rays) flux intensities they have to be coupled in a consistent manner to the other differential equations describing dynamics and thermodynamics of the low-energy plasma constituents.

We describe the time-dependent response of the whole interface system considering the imprint of the solar-cyclically varying dynamical solar wind pressure at the inner border within a fully-time-dependent htdrodynamic-simulation of the five dynamically relevant, interacting solar and interstellar fluids. Hereby we follow VOAYGER-1/2 data taken by Gazis (1994) and try to best-fit them by the following analytic time-dependencies: For the solar wind bulk flow veloc- ity $V_{s}$ we adopt (for details see Scherer and Fichtner, 2004):

$$
\begin{aligned}
& f(t))=a_{s}+b_{s} \cos \left(\omega_{s} t\right) \exp \left[\cos \left(\omega_{s} t\right)\right] \\
& V_{s}(t)=V_{\min }+\Delta V_{s} f(t)
\end{aligned}
$$

with the solar cycle period $\tau_{s}=2 \pi / \omega_{s}=11 \mathrm{yrs}$, and with $V_{\min }=300 \mathrm{~km} / \mathrm{s}$ and $\Delta V_{s}=v_{\max }-v_{\min }=500 \mathrm{~km} / \mathrm{s}$. The constants $a_{s}, b_{s}$ are chosen to normalize the function $f$ to values between 0 and 1, e.g. $a_{s}=b_{s} e^{-1}$ and $b_{s}=1 /\left(e^{1}+e^{-1}\right)$. As discussed in Scherer and Fahr (2003a) and Scherer and Fichtner (2004) the solar wind mass flow $\Phi_{m s}$ is constant (see also McComas et al., 2004) and hence the proton density anticorrelates with the bulk velocity like:

$n_{s}(t)=\frac{\Phi_{m s}}{V_{\min }+\Delta V_{s} f(t)}$

Therefore, the most relevant dynamical quantity, the dynamical pressure $\Pi_{s}$ at the inner boundary of our simulation program is given by:

$\Pi_{s}(t)=\Phi_{m s}\left[V_{\min }+\Delta V_{s} f(t)\right]$

Two quantities, i.e. $V_{s}(t)$ and $n_{s}(t)$ are plotted as function of the solar cycle phase time $t$ in Fig. (3), while the third quantity $\Pi_{s}(t)$ behaves analogously to the solar wind speed.

We now briefly describe the time-dependent dynamical and thermodynamical coupling of these low- and high- energy plasma components to the above time-variable inner boundary conditions: The first-order interaction of the solar wind with the interstellar plasma is a hydrodynamic proton - proton interaction. In addition to protons, however, also interstellar $\mathrm{H}$-atoms are flying into the heliosphere which upon ionization in the region of the supersonic solar wind produce H-pick-up ions (PUI's). Due to effective pitch angle scattering they are rapidly isotropized in velocity space and are comoving with the solar wind, thereby representing a KeV-energetic ion load of the expanding solar wind. These PUI's are treated in our model as an additional ion fluid, separate from the solar wind proton fluid by its temperature, its pressure and its number density. A certain fraction of these PUI's upon arrival at the termination shock can be injected into the Fermi-1 diffusive acceleration process (see Chalov and Fahr, 1997) and act as a seed for the ACR population which in our HD- multifluid model is also treated by its relevant fluid moments, i.e. the energy density or pressure. By the ACR pressure gradient this high energy fluid is interacting with the solar wind plasma flow and for instance decelerates this flow in the region near and upstream of the termination shock forming a shock precursor. For time-dependent conditions the physics in this precursor for the convected low energy plasma species must be evaluated for the event of time $t$ counted by the computer, i.e. the following flow conservation laws (see Chalov and Fahr, 1994, 1995, 1997) have to be 
fulfilled at $t$ :

$$
\begin{gathered}
\nabla \cdot\left(\rho_{i} \boldsymbol{v}_{s}\right)=Q_{\rho i} \\
\nabla \cdot\left(\rho \boldsymbol{v}_{s} \boldsymbol{v}_{s}+\left(P_{s}+P_{\mathrm{pui}}+P_{\mathrm{ACR}}+P_{\mathrm{GCR}}\right) \overleftrightarrow{I}\right)=\boldsymbol{Q}_{p} \\
\nabla \cdot\left(\rho \boldsymbol{v}_{s}\left\{\frac{v_{s}^{2}}{2}+\frac{\gamma}{\gamma-1} \frac{1}{\rho}\left(P_{s}+P_{\mathrm{pui}}\right)\right\}\right. \\
\left.+\boldsymbol{\Phi}_{\boldsymbol{C R}}\right)=Q_{e}
\end{gathered}
$$

where $\rho=\rho_{s}+\rho_{\text {pui }}$ denotes the total mass density of the mixed fluids. The quantities $P_{s}, P_{\text {pui }}, P_{\mathrm{ACR}}, P_{\mathrm{GCR}}$ represent the pressures of the solar wind protons, the PUI's, the ACR's and the GCR's, respectively. The polytropic index $\gamma=5 / 3$ is taken to be valid for protons and PUI's as well, and the quantities $Q_{\rho i}, \boldsymbol{Q}_{p}, Q_{e}$ denote mass-, momentum-, and energy exchange rates per unit volume and time. The contribution to the energy flow of the coupled ACR's and GCR's is separately denoted by $\boldsymbol{\Phi}_{C R}=\boldsymbol{\Phi}_{\mathrm{ACR}}+\boldsymbol{\Phi}_{\mathrm{GCR}}$ and is itself given by the following differential equation:

$$
\begin{aligned}
& \mathbf{\Phi}_{\mathrm{ACR}}=\frac{\gamma_{\mathrm{ACR}}}{\gamma_{\mathrm{ACR}}-1} \boldsymbol{v}_{s} P_{\mathrm{ACR}}-\frac{\kappa_{\mathrm{ACR}}}{\gamma_{\mathrm{ACR}}-1} \nabla P_{\mathrm{ACR}} \\
& \mathbf{\Phi}_{\mathrm{GCR}}=\frac{\gamma_{\mathrm{GCR}}}{\gamma_{\mathrm{GCR}}-1} \boldsymbol{v}_{s} P_{\mathrm{GCR}}-\frac{\kappa_{\mathrm{GCR}}}{\gamma_{\mathrm{GCR}}-1} \nabla P_{\mathrm{GCR}}
\end{aligned}
$$

where $\gamma_{\mathrm{ACR} / \mathrm{GCR}}=4 / 3$ and $\kappa_{\mathrm{ACR} / \mathrm{GCR}}$ are the polytropic indices of the high-energy ACR/GCR components and the energy-averaged ACR/GCR spatial diffusion coefficient. Both ACR's and GCR's are treated as massless fluids by separate energy-averaged particle transport equations. The coupling of these massless fluids occurs due to convective interactions of the ACR/GCR fluids with the background plasma, inducing a modification of the plasma flow by ACR and GCR pressure gradients. The required consistency within this model must also include the dynamical and thermodynamical coupling of the mentioned four fluids to one more fluid, namely the $\mathrm{H}$-atoms. In our model we treat the $\mathrm{H}$-atoms as an additional hydrodynamical fluid coupled by charge exchange reactions to protons and PUI's. The complete modelling within a stationary two-dimensional multifluid simulation is explained in detail by Fahr (2000) and Fahr et al. (2000), the so-called five-fluid Bonn model. The time-dependent version of this model was presented by Scherer and Fahr (2003a,b) and in fact delivers all the time-dependent ingredients which are needed to calculate the above mentioned spectral ENA fluxes.

\section{Inclusion of PUI removals}

In Sect. 5 we have derived the PUI distribution function starting out from the PUI transport equation. The solution for the PUI distribution function downstream of the termination shock is given by Eq. (14) and contains a local values of an extinction function describing accumulated PUI losses due to charge exchange reactions of pick-up ions while being convected with the solar wind plasma flow downstream from the
TS-shock through the heliosheath to the local point $r$ where $f_{\text {pui }}$ is required. This extinction function contains a specific convection time $\tau(s(\theta))$ which can not easily be calculated for arbitrary places in the heliosheath. For our calculations presented above this extinction function has been set equal to 1 at all places $r$ and the distribution function $f_{\text {pui }}^{\text {sheat was }}$ approximated by the ratio of the PUI number densities at the shock and at a distance $r$, see Eq. (16). This is why we have to call our below presented PUI-induced ENA fluxes as upper limits, since the realistically resulting extinction losses would in fact reduce the resulting ENA fluxes which are to be expected at Earth. By how much this reduction would occur strongly depends on the source place $r$ where the ENA particle is produced and on the energy of the PUI from which the ENA is produced by $\mathrm{H}$-atom charge exchange.

In the following we want to give estimates for these extinction losses and for the resulting ENA flux reductions that can be expected. For that purpose we consider losses of PUI's convected with the solar wind flow along the streamlines which originate at the upwind portion of the termination shock at the PUI convection along the stagnation line towards the stagnation point near the upwind heliopause nose. First we calculate within the frame of our multifluid interface model the convection times $\left.\tau(s(\theta=0))=\tau\left(s_{0}\right)\right)$ using the informations at the grid points $s_{i}$ :

$\tau\left(s_{0}\right)=\int_{0}^{s_{0}} \frac{d s}{v_{s}(s)}=\sum_{i=1}^{i=i_{0}} \frac{\Delta s_{i}}{v_{s}\left(s_{i}\right)}$

These convection times are identical for all PUI energies $w$. The extinction losses, however, are energy-sensitive and given by.

$\Lambda\left(s_{0}, w\right)=\exp \left[-n_{H}\left(s_{0}\right) \sigma_{\mathrm{ex}}(w) V_{s} \sqrt{w} \tau\left(s_{0}\right)\right]$

where $n_{H}\left(s_{0}\right)$ is the $s$ - dependent LISM H-atom density in the inner heliosheath as it results from our multifluid interface model, and where the energy-dependent charge exchange cross section is given by:

$\sigma_{\mathrm{ex}}(w)=\left[A-B \log \left(V_{s}^{*} \sqrt{w}\right)\right]^{2}$

with $A=1.6 \cdot 10^{-7}$ and $B=6.8 \cdot 10^{-9}$, and $V_{s}^{*}$ being the upstream solar wind bulk velocity in units of $[\mathrm{cm} / \mathrm{s}]$.

In Fig. 7 we show the extinction function $\Lambda\left(s_{0}, w\right)$ as function of $s_{0}$ for various values of the PUI's of different energies convected outwards from the termination shock in downstream direction along the stagnation line. As one can see there for large energies the function is rapidly decreasing with distance $s_{0}$ downstream of the TS-shock, whereas for small energies it decreases much less rapidly. This behavior is a direct consequence of Eq. (27) showing the dependence of the extinction function on the particle velocity $v$ or energy $w$ via the product of the charge exchange cross section and the mean relative velocity $\sqrt{w}$ between these PUI's and $\mathrm{H}$-atoms. Since this relative velocity is practically identical with the PUI velocity $\sqrt{w}$, and since the cross section only decreases by the logarithm of the PUI velocity (see Eq. (28), 


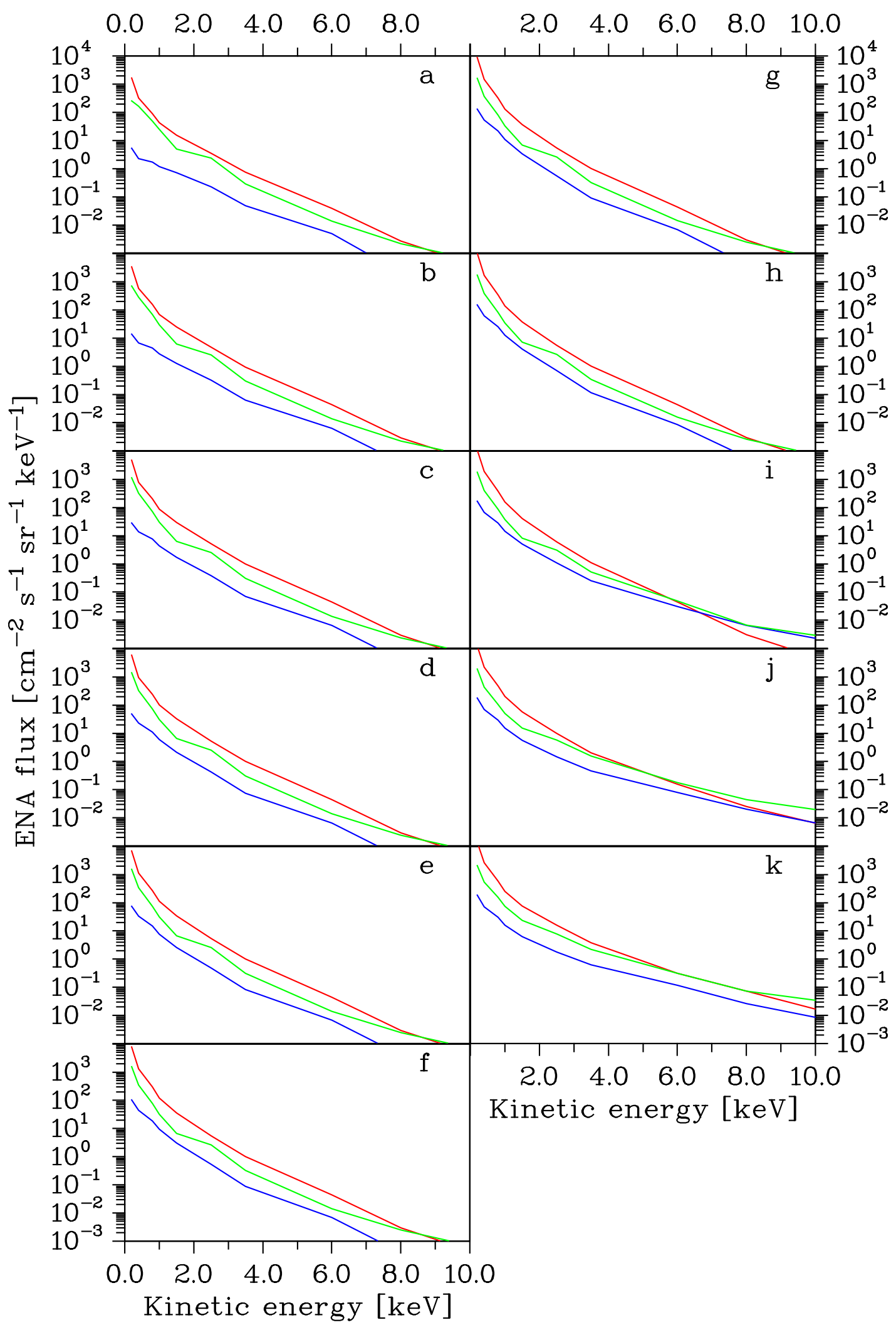

Fig. 4. The ENA fluxes from the upwind direction, integrated along the line of sight. The red lines show the ENA-fluxes from shocked solar wind protons, the green lines show the flux of the secondary ENAs build from PUIs between the termination shock and the heliopause, and the blue lines show the foreground PUIs created in the supersonic solar wind. The labels a to k correspond the labels shown below the $x$-axis in Fig. (3) and correspond to a 1 year time-step between two consecutive panels, starting from the declining solar cycle (see Fig. (3)). 


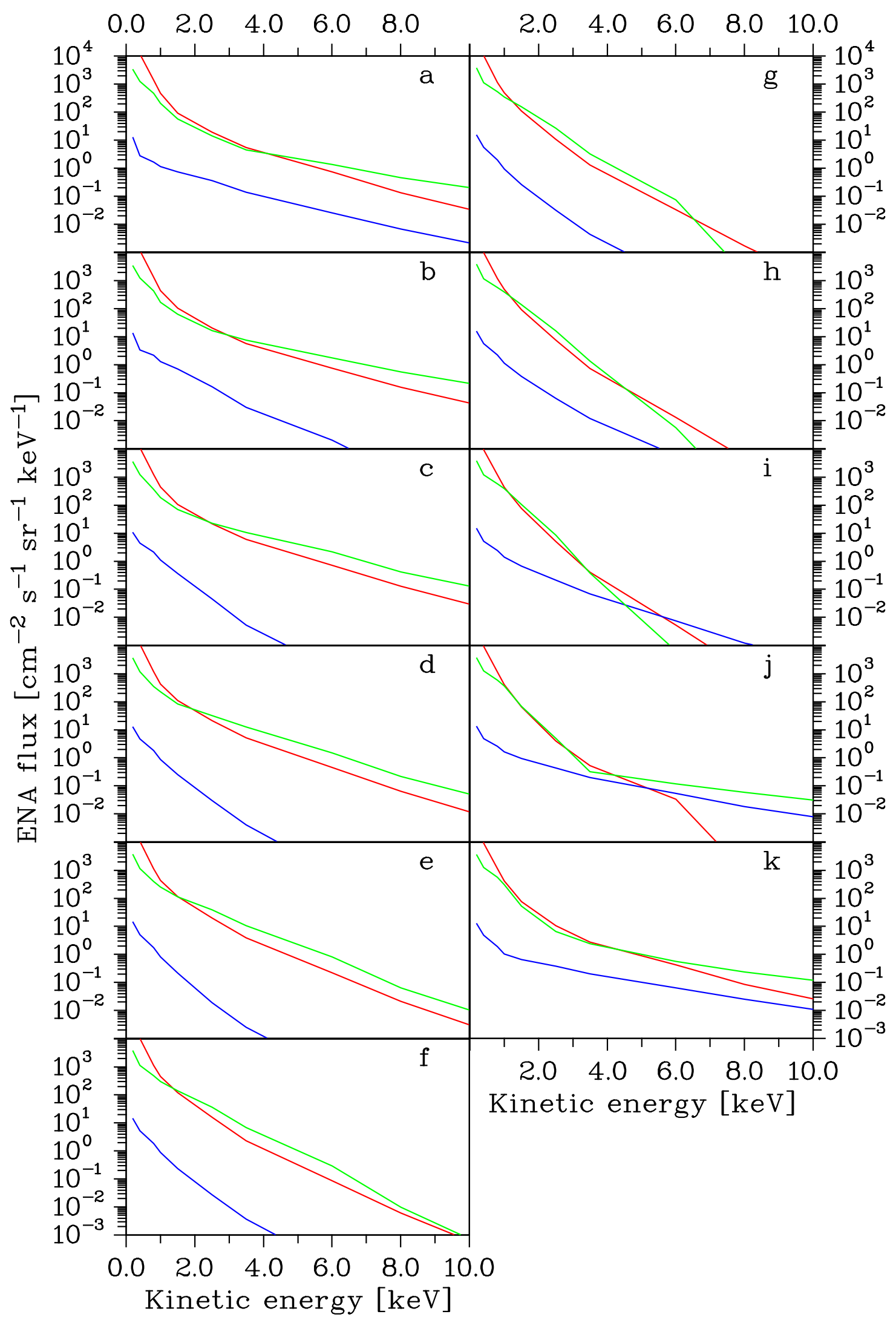

Fig. 5. The same as in Fig. 4, for the fluxes from the downwind direction. 


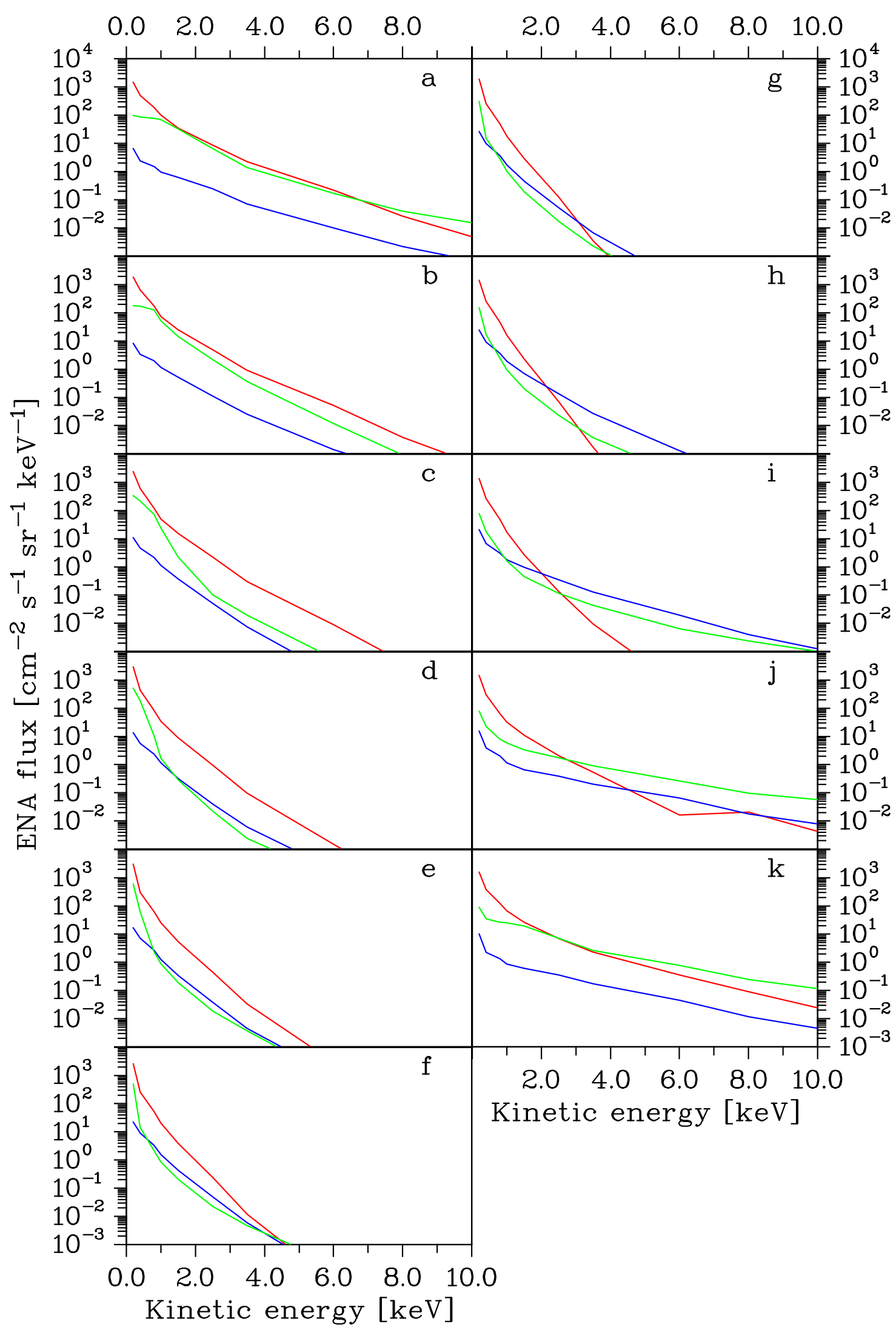

Fig. 6. The same as in Fig. 4 for the fluxes from the crosswind direction. 


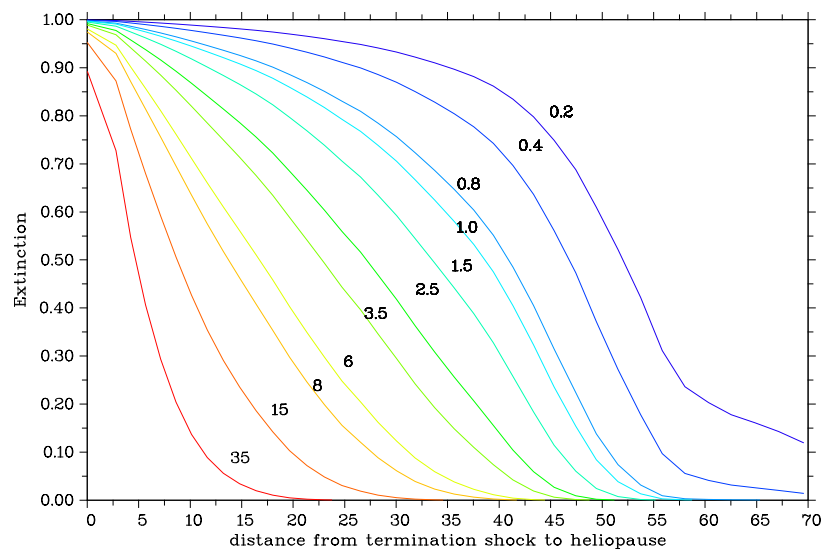

Fig. 7. The extinction of PUI-ENAs along the stagnation line for different energies. The labels on the curves indicates the kinetic energy of the PUIs in $\mathrm{KeV}$, see also Eq. (27).

this dependence thus leads to higher extinction rates for increasing PUI velocities. This may tell that PUI-ENA's with high energies are collected from regions relatively close to the TS-shock, whereas PUI-ENA's with low energies are collected from a much more extended heliosheath region. This fact may also be taken as a diagnostic tool to study the extent of the heliosheath and the distance to the stagnation point.

\section{Discussion of the results}

In Figs. (4) to (6) we have shown the expected spectral fluxes at earth of ENA's of different origins, i.e. decharged PUI's of the inner heliosphere (blue curves), decharged protons of the outer heliosphere (red curves) and decharged PUI's of the outer heliosphere (green curves). Figure (4) displays fluxes reaching the earth from the upwind direction, Fig. (5) those from the downwind direction, and Fig. (6) those from the crosswind direction. Each of these Figures are split into 11 separate diagrams showing actually resulting flux conditions realized over 11 consecutive years within the solar activity cycle, i.e. "a" indicates the beginning of a solar cycle, and " $k$ " the end of the cycle. The corresponding inner solar wind boundary conditions for the diagrams " $\mathrm{a}$ " to " $\mathrm{k}$ " can be read out from the Fig. (3) displaying the solar cycle - induced variation of the solar wind velocity, density and dynamic pressure.

Since the whole heliospheric interface is in a fully timedependent response to the time-variable inner solar wind boundary conditions the fluxes of ENA's of different origin also react strongly time-dependent, however, the different ENA species in substantially different forms. The ENA fluxes from upwind up to some critical energy $E_{c \text {,up }}$ are dominated by ENA's due to decharged protons downstream of the termination shock, while beyond that energy $E_{c, \text { up }}$ they are dominated by ENA's due to decharged PUI's downstream of the termination shock. The first types of ENA's were already treated in an earlier paper by Gruntman et al. (2001) who simply had taken a solar wind termination shock at $90 \mathrm{AU}$ and had calculated ENA fluxes for different shock compression ratios. The realistic situation, however, is much more complicate, since the whole heliospheric plasma interface under the action of the varying inner solar dynamic pressure reacts fully-time dependent placing during the consecutive phases of the solar cycle the TS shock at different distances with varying compression ratios. This critical energy $E_{c \text {,up }}$ in upwind direction is always found as $E_{c \text {,up }}>5 \mathrm{KeV}$, increasing up to $E_{c \text {,up }}=10 \mathrm{KeV}$ during some phases of the solar activity cycle (panels $\mathrm{i}, \mathrm{j}, \mathrm{k}$ in Fig. 4). In all phases, labeled by "a" through " $k$ ", the flux contributions from ENA's due to decharged PUI's in the inner heliosphere are negligible.

In downwind direction this situation is different and more complicated (see Fig. (5)). The critical energy $E_{c \text {,down }}$ here only is of the order of $E_{c \text {,down }} \leq 2 \mathrm{KeV}$, but fluxes of ENA's due to decharged PUI's in the inner heliosphere may even become dominant here during some phases of the solar activity cycle. Similar results can be found in Fig. (6) displaying the fluxes from the crosswind direction. This all confirms that not only ENA fluxes are highly variable in time over the solar activity cycle, their relative contributions also varies very much with looking direction. The best way to disentangle the different ENA contributions is to look in upwind direction at energies above $E_{c \text {,up }}$ (i.e. ENA's due to decharged PUI's downstream of the termination shock) or energies below $E_{c \text {,up }}$ (i.e. ENA's due to decharged protons downstream of the termination shock), or to look into the downwind direction at some phase of the solar cycle for ENA's from decharged PUI's in the inner heliosphere.

While in the upwind-direction the fluxes of both populations are ordered in time in the same way, in the crosswind direction the order is not correlated. This is due to the fact, that the crosswind sheath represents a mixture of shocked solar wind from the crosswind direction and wind shocked on all positions from the upwind direction onward. This flow needs some time to pass from the upwind to the crosswind direction and is mixed up from all directions in between. Therefore, one cannot gain much information on the interface from the crosswind direction.

In the downwind direction the situation again has changed, because no flow lines from crosswind will pass into the tail region. The solar cycle induced changes are still dominating in most phases of the solar cycle, but now also the large volume of the tail region plays a crucial role for the production of secondary slow ENAs. Therefore, the contribution form the slow ENAs dominates above a low critical energy $E_{c}<2 \mathrm{KeV}$. Because also in the downwind direction, the solar cycle variations of previous cycles are "memorized" in the neutral hydrogen flux, at higher energies the contribution from the fast ENAs can be dominant during some phases of the solar cycle.

To distinguish between the ENA background produced in the supersonic solar wind from the flux coming from the interface, is possible in the upwind- and downwind direction for most phases of the solar cycle, but becomes critical for the crosswind direction. This fact should be kept in mind in designing future space-probes mission profiles. 
Acknowledgement. The authors are grateful for financial support granted to them by the Deutsche Forschungsgemeinschaft in the frame of the project "Heliotrigger" (Fa 97/28-1).

Edited by: B. Heber

Reviewed by: S. Barabash and another referee

\section{References}

Burlaga, L. F., Ness, N. F., Stone, E. C., McDonald, F. B., Acuna, M. H., Lepping, R. P., and Connerney, J. E. P.: Search for the heliosheath with Voyager 1 magnetic field measurements, Geopyhs. Res. Lett., 30, 2072, DOI 10.1029/2003GL018291, 2003.

Burch, J. L.: The first two years of IMAGE, Space Sci. Rev., 109, 1-24, 2003.

Chalov, S. V. and Fahr, H.-J.: A two-fluid model of the solar wind termination shock modified by shock-generated cosmic rays including energy losses, Astron. Astrophys., 288, 973-980, 1994.

Chalov, S. V. and Fahr, H.-J.: ntropy generation at the multi-fluid solar wind termination shock producing anomalous cosmic ray particles, Planet. Space Sci., 43, 1035-1043, 1995.

Chalov, S. V. and Fahr, H.-J.: The three-fluid structure of the particle modulated solar wind termination shock, Astron. Astrophys., 326, 860-869, 1997.

Chalov, S. V. and Fahr, H.-J.: Phase space diffusion and anisotropic pick-up ion distributions in the solar wind: an injection study, Astron. Astrophys., 335, 746-756 1998.

Chalov, S. V., Fahr, H.-J., and Izmodenov, V.: Evolution of pickup proton spectra in the inner heliosheath and their diagnostics by energetic neutral atom fluxes, J. Geophys. Res., 108, 11-1, DOI:10.1029/2002JA009492, 2003

Chalov, S. V., Fahr, H.-J., and Izmodenov, V.: Spectra of energized pick-up ions upstream of the two-dimensional heliospheric termination shock. II, Acceleration by Alfvenic and by large-scale solar wind turbulences, Astron. Astrophys., 320, 659-671, 1997.

Chalov, S. V., Fahr, H.-J., and Izmodenov, V.: Spectra of energized pick-up ions upstream. of the heliospheric termination shock I. The role of Alfvenic turbulences, Astron. Astrophys., 304, 609616, 1995.

Czechowski, A., Fichtner, H., Grzedzielski, S., Hilchenbach, M., Hsieh, K. C., Jokipii, J. R., Kausch, T., Kota, J., and Shaw, A.: Anomalous cosmic rays and the generation of energetic neutrals in the region beyond the termination shock, Astron. Astrophys., 368, 622-634, 2001a.

Czechowski, A., Fahr, H.-J., Lay, G., and Hilchenbach, M.: Pick-up ions upstream and downstream of the termination shock, Astron. Astrophys., 379, 601-610, 2001b.

Fahr, H.-J.: The Multifluid Character of the 'Baranov' Interface, Astrophys. Space Sci., 274, 35-54, 2000.

Fahr, H.-J. and Lay, G.: Remote diagnostic of the heliospheric termination shock using neutralized post shock pick-up ions as messengers, Astron. Astrophys., 356, 327-334, 2000.

Fahr, H.-J., Kausch, T., and Scherer, H.: A 5-fluid hydrodynamic approach to model the solar system-interstellar medium interaction, Astron. Astrophys., 357, 268-282 , 2000.

Gazis, P. R.: Pioneer and Voyager observations of solar cycle variations in the outer heliosphere, Geophys. Res. Lett., 21, 17431746, 1994.

Gruntman, M.: Energetic neutral atom imaging of space plasmas, Rev. Sci. Inst., 68, 3617-3656, 1997.
Gruntman, M., Roelof, E. C., Mitchell, D. G., Fahr, H.-J., Funsten, H. O., and McComas, D. J.: Energetic neutral atom imaging of the heliospheric boundary region, J. Geophys. Res., 106, 15767-15 782, 2001.

Hilchenbach, M., Hsieh, K. C., Hovestadt, D., Klecker, B., Gruenwaldt, H., Bochsler, P., Ipavich, F. M., Buergi, A., Moebius, E., Gliem, F., Axford, W. I., Balsiger, H., Bornemann, W. Coplan, M. A., Galvin, A. B., Geiss, J., Gloeckler, G., Hefti, S., Judge, D. L., Kallenbach, R., Laeverenz, P., Lee, M. A., Livi, S., Managadze, G. G., Marsch, E., Neugebauer, M., Ogawa, H. S., Reiche, K.-U., Scholer, M., Verigin, M. I., Wilken, B., and Wurz, P.: Detection of $55-80 \mathrm{keV}$ Hydrogen Atoms of Heliospheric Origin by CELIAS/HSTOF on SOHO, Astrophys. J., 503, 916, 1998.

Izmodenov, V. V.: Hot Neutral $\mathrm{H}$ in the Heliosphere: Elastic H-H, H-p Collisions, Astrophys. Space Sci., 274, 71-76, 2000.

Izmodenov, V. V. and Malama, V.: Variations of interstellar $\mathrm{H}$ atom parameters in the outer heliosphere: solar cycle effects, Adv Space Res., 34, 74-78, 2004.

Kausch, T. and Fahr, H.-J.: Interstellar gas filtration to the inner heliosphere under the selfconsistent influence of a pick-up ion modulated termination shock, Astron. Astrophys., 325, 828-838, 1997.

LeRoux, J. A. and Fichtner, H.: A self-consistent determination of the heliospheric termination shock structure in the presence of pickup, anomalous, and galactic cosmic ray protons, J. Geophys. Res., 102, 17365-17 380, 1997.

Krimigis, S. M., Decker, R. B., Hill, M. E., Armstrong, T. P., Gloeckler, G., Hamilton, D. C., Lanzreotti, L. J., and Roelof, E. C.: Voyager 1 exited the solar wind at a distance of 85 AU from the Sun, Nature, 426, 45-48, 2003.

McComas, D., Allegrini, F., Bochsler, P., Bzowski, M., Collier, M., Fahr, H., Fichtner, H., Frisch, P., Funsten, H., Fuselier, S., Gloeckler, G., Gruntman, M., Izmodenov, V., Knappenberger, P., Lee, M., Livi, S., Mitchell, D., Möbius, E., Moore, T., Reisenfeld, D., Roelof, E., Schwadron, N., Wieser, M., Witte, M., Wurz, P., and Zank, G.: The Interstellar Boundary Explorer (IBEX), AIP Conf. Proc. 719: Physics of the Outer Heliosphere, 162-181, 2004

McDonald, F. B., Stone, E. C., Cummings, A. C., Heikkila, B., Lal, N., and Webber, W. R.: Enhancements of energetic particles near the heliospheric termination shock, Nature, 426, 48-51, 2003.

McKenzie, J. F. and Westphal, K. O.: Transmission of Alfvén waves through the earth's bow shock, Planet. Space Sci., 17, 10291037, 1969.

Scherer, K. and Fahr, H.-J.: Solar cycle induced variations of the outer heliosphere structures, Geophys. Res Let., 29, DOI: 10.1029/2002GL016073, 2002.

Scherer, K. and Fahr, H.-J.: Breathing of heliospheric structures triggered by the solar-cycle activity, Ann. Geophys., 21, 1303 1313, 2003a, Sref-ID: 1432-0576/ag/2003-21-1303.

Scherer, K. and Fahr, H.-J.: Remote diagnostic of the solar-cycleinduced heliospheric interface variation using energetic neutral atoms, Astron. Astrophys. 404, L47-L50, 2003 b.

Scherer, K. and Fichtner, H.: Constraints on the heliospheric magnetic field variation during the Maunder Minimum from cosmic ray modulation modelling, Aston. Astrophys., 413, L11-L14, 2004.

Williams, D. J., Roelof, E. C., and Mitchell, D. G.: Global magnetospheric imaging, Rev. Geophys., 30, 183-208, 1992. 
Zank, G. P., Webb, G. M., and Donohue, D. J.: Particle injection and the structure of energetic-particle-modified shocks, Astrophys. J., 406, 67-91, 1993.

Zank, G. P.: Interaction of the solar wind with the local interstellar medium: a theoretical perspective, Space Sci. Rev., 89, 413-688, 1999.

Zank, G. P. and Müller, H.-R.: The dynamical heliosphere, J. Geophys. Res., 108, SSH 7-1, doi: 10.1029/20002JA009689, 2003. 